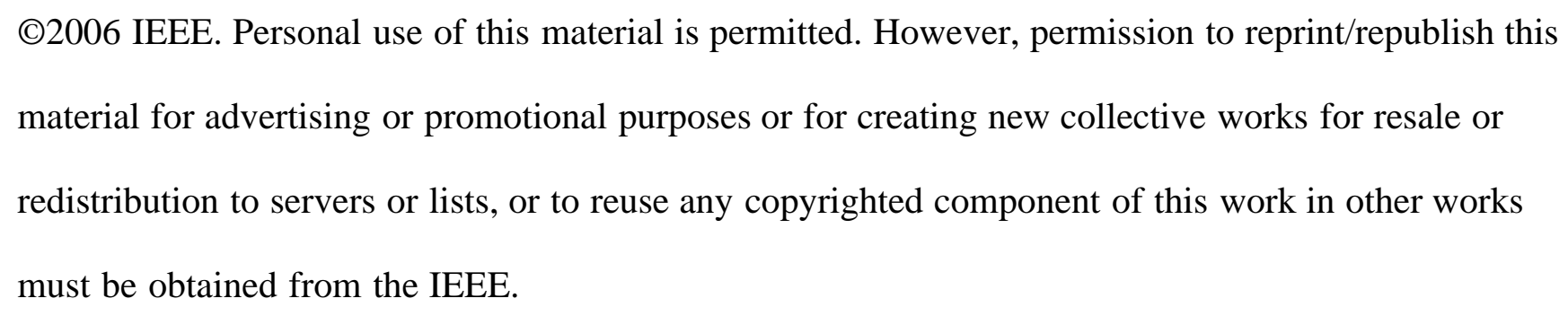




\section{Performance Tradeoffs among Beamforming Approaches}

\author{
Yue Rong \\ University of California, Riverside \\ Riverside, CA 92521, USA
}

\author{
Yonina C. Eldar \\ Technion, Israel Institute of Technology \\ Haifa, Israel 32000
}

\author{
Alex B. Gershman \\ Darmstadt University of Technology \\ 64283 Darmstadt, Germany
}

\begin{abstract}
In this paper, three main criteria for the adaptive beamformer design are discussed: maximal signal-tointerference-plus-noise ratio (MSINR), minimal mean-squared error (MMSE), and minimal least-square error (MLSE). Although in the case of exactly known power and steering vector of the signal-of-interest (SOI), there are beamformers that can simultaneously meet the MMSE and MSINR criteria, this is no longer true when the exact knowledge of the steering vector is unavailable. To account for steering vector errors, a meaningful approach is to model the actual steering vector as random. In this paper, it is shown that in the latter case, the MMSE and MSINR criteria can not be simultaneously attained. We study the achievable region in the MSE-SINR plane and propose a new adaptive beamformer that can attain a frontier of operating points on the boundary of this region and, therefore, provide an optimal performance tradeoff among the MSINR and MMSE criteria. It is also shown that in the random steering vector case, the MLSE and MSINR criteria are simultaneously achievable and a new adaptive beamformer is proposed that satisfies both these criteria.
\end{abstract}

\section{INTRODUCTION}

In many applications such as radar and sonar, the main criterion for beamformer design is to maximize the output signal-to-interference-plus-noise ratio (SINR). This class of beamformers is called maximal SINR (MSINR) techniques [1]. However, maximizing SINR does not necessarily guarantee an acceptably good estimate of the signal waveform [2]. Note that in many applications, it is more important to minimize the signal estimation error rather than maximize the SINR. For example, in digital communications the quality of estimating the amplitude and phase of the received SOI is crucial for the detection of the information-bearing symbols. In such cases, minimal mean-squared error (MMSE) beamforming is of great interest [2], [3]. Unfortunately, the signal power needs to be known for the MMSE beamformer design, and in many practical applications this knowledge may be difficult to obtain. To avoid this difficulty, minimal least-square error (MLSE) beamformers can be applied [2]. MLSE beamformers are based on minimizing the LSE of the array observations, and do not require any knowledge of the signal power.

An "ideal" beamforming approach should meet both the MSINR and MMSE criteria, whenever possible. It is a well known fact that if the SOI power and the steering vector are known exactly, the MMSE beamformer also maximizes the output SINR. If the signal power is unknown, then the MLSE beamformer can be alternatively used. The latter beamformer not only maximizes the SINR, but also asymptotically minimizes the MSE in the high signal power case.

In practical applications, due to different reasons such as signal pointing errors, imperfect array calibration, and environmental nonstationarities, errors between the actual and the presumed steering vectors may occur [4]-[10]. A popular approach to account for such errors is to model the actual steering vector as random with known distribution [5]-[8]. In our paper, we assume that the actual steering vector is Gaussian with known mean and covariance matrix. As will be seen later, in this case the MMSE beamformer does not maximize the output SINR. That is, the MMSE and MSINR criteria can not be simultaneously achieved.

For the convenience of the following discussion, let us refer to a plane whose two coordinates represent the values of MSE and SINR as the MSE-SINR plane. In fact, any beamformer is associated with a certain point on this plane. In the case of a random steering vector, we show that not all points on the MSE-SINR plane are achievable. Clearly, such beamformers are of interest that yield small MSE and large SINR, thereby achieving a proper MSE-SINR tradeoff.

With such a motivation, a new beamformer is proposed that provides an optimal MSE-SINR tradeoff by minimizing the MSE subject to a given SINR constraint. In terms of the MSESINR plane, the points corresponding to the proposed beamformer form an optimal tradeoff curve which is the frontier that divides the whole MSE-SINR plane into two parts that contain achievable and non-achievable points, respectively.

The knowledge about the optimal tradeoff between the MSE and SINR is quite important for practical applications where the objective is to optimize a combination of these two criteria. In what follows, it will be shown that such a beamformer can be easily obtained using the optimal tradeoff curve.

As mentioned above, in the case of unknown signal power the MLSE beamformer can be used instead of the MMSE beamformer. It is proven that in the considered case of random steering vector, the MLSE and MSINR criteria can be simultaneously achieved and a new beamformer is proposed that achieves both these criteria.

\section{BACKGROUND}

The $M \times 1$ observation vector of an $M$-sensor antenna array at the time $t$ is given by

$$
\mathbf{y}(t)=s(t) \mathbf{a}+\mathbf{i}(t)+\mathbf{v}(t)
$$


where $\mathbf{a}$ is the SOI steering vector, $s(t)$ is the SOI waveform, and $\mathbf{i}(t)$ and $\mathbf{v}(t)$ are the interference and noise vectors, respectively. In the sequel, we will omit the dependence of $t$ for notational simplicity.

In many practical applications, it is difficult to obtain the information about the actual steering vector because of pointing errors, imperfect array calibration, environmental nonstationarities, etc. Many authors addressed the problem of robust beamformer design when only a partial information about the actual steering vector is available [4]-[10]. In this paper, we consider the scenario where the SOI steering vector is complex Gaussian distributed [5]-[7] with the mean $\mathbf{m}$ and the covariance matrix $\mathbf{C}$, that is, $\mathbf{a} \sim \mathcal{C N}(\mathbf{m}, \mathbf{C})$. In particular, the mean vector $\mathbf{m}$ corresponds to the perturbationfree steering vector, while the covariance matrix $\mathbf{C}$ captures uncertainties in this vector. We assume that $\mathbf{m}$ and $\mathbf{C}$ are known and that $\mathbf{C} \succ 0$.

The output of a narrow-band beamformer is given by

$$
\hat{s}=\mathbf{w}^{H} \mathbf{y}
$$

where $\hat{s}$ is the estimated signal waveform, w is the $M \times 1$ weight vector, and $(\cdot)^{H}$ stands for the Hermitian transpose.

The MSINR beamformer aims at maximizing the output SINR. In the case of random steering vector, the expectation of the output SINR with respect to $\mathbf{a}$ is given by

$$
\mathrm{SINR}=\mathrm{E}_{\mathbf{a}}\left\{\frac{\sigma_{s}^{2}\left|\mathbf{w}^{H} \mathbf{a}\right|^{2}}{\mathbf{w}^{H} \mathbf{R}_{i+n} \mathbf{w}}\right\}=\frac{\sigma_{s}^{2} \mathbf{w}^{H} \mathbf{R}_{a} \mathbf{w}}{\mathbf{w}^{H} \mathbf{R}_{i+n} \mathbf{w}}
$$

where $\sigma_{s}^{2}$ denotes the signal power, $\mathbf{R}_{i+n}=\mathrm{E}\{(\mathbf{i}+\mathbf{v})(\mathbf{i}+$ $\left.\mathbf{v})^{H}\right\}$ represents the interference-plus-noise covariance matrix, $\mathbf{R}_{a}=\mathrm{E}_{\mathbf{a}}\left\{\mathbf{a a}^{H}\right\}=\left(\mathbf{C}+\mathbf{m} \mathbf{m}^{H}\right)$ is the correlation matrix of the steering vector, and $\mathrm{E}_{\mathbf{a}}\{\cdot\}$ denotes the statistical expectation with respect to the random vector $\mathbf{a}$. Maximizing (1) with respect to $\mathbf{w}$, the weight vector of the MSINR approach can be written as [1]

$$
\mathbf{w}_{\text {MSINR }}=\alpha \mathcal{P}\left\{\mathbf{R}_{i+n}^{-1} \mathbf{R}_{a}\right\}
$$

where $\alpha$ is an arbitrary nonzero scalar and $\mathcal{P}\{\cdot\}$ stands for the principal eigenvector of a matrix. A common approach to choose $\alpha$ is to use the minimum variance distortionless response (MVDR) formulation of the MSINR problem:

$$
\min _{\mathbf{w}} \mathbf{w}^{H} \mathbf{R}_{i+n} \mathbf{w} \quad \text { s.t. } \quad \mathbf{w}^{H} \mathbf{R}_{a} \mathbf{w}=1 .
$$

According to (3), the scalar $\alpha$ in (2) is given by

$$
\alpha=\left(\mathcal{P}\left\{\mathbf{R}_{i+n}^{-1} \mathbf{R}_{a}\right\}^{H} \mathbf{R}_{a} \mathcal{P}\left\{\mathbf{R}_{i+n}^{-1} \mathbf{R}_{a}\right\}\right)^{-1 / 2} .
$$

Substituting (2) back into (1), the maximal output SINR can be expressed as

$$
\operatorname{SINR}_{\mathrm{o}}=\sigma_{s}^{2} \lambda\left\{\mathbf{R}_{i+n}^{-1} \mathbf{R}_{a}\right\}
$$

where $\lambda\{\cdot\}$ stands for the maximal eigenvalue of a matrix.

As mentioned above, maximizing the output SINR does not necessarily lead to a good estimate of the signal waveform, and in some cases it is more important to have the lowest waveform estimation error than the highest SINR. In such cases, the MMSE beamformer can be used. The MSE of the signal waveform estimation for one realization of the steering vector $\mathbf{a}$ is given by

$$
\mathrm{E}\left\{|\hat{s}-s|^{2}\right\}=\sigma_{s}^{2}\left|1-\mathbf{w}^{H} \mathbf{a}\right|^{2}+\mathbf{w}^{H} \mathbf{R}_{i+n} \mathbf{w}
$$

where the expectation $\mathrm{E}\{\cdot\}$ is defined with respect to the random noise, interference, and the signal waveform. Using (6), the expectation of (6) with respect to $\mathbf{a}$ is given by

$$
\begin{aligned}
\mathrm{MSE} & =\mathrm{E}_{\mathbf{a}}\left\{\sigma_{s}^{2}\left|1-\mathbf{w}^{H} \mathbf{a}\right|^{2}+\mathbf{w}^{H} \mathbf{R}_{i+n} \mathbf{w}\right\} \\
& =\mathbf{w}^{H}\left(\mathbf{R}_{i+n}+\sigma_{s}^{2} \mathbf{R}_{a}\right) \mathbf{w}-2 \operatorname{Re}\left\{\sigma_{s}^{2} \mathbf{w}^{H} \mathbf{m}\right\}+\sigma_{s}^{2}(7)
\end{aligned}
$$

where $\operatorname{Re}\{\cdot\}$ denotes the real part. The MMSE beamformer weight vector is obtained by minimizing (7) and can be expressed as [2]

$$
\mathbf{w}_{\mathrm{MMSE}}=\sigma_{s}^{2}\left(\mathbf{R}_{i+n}+\sigma_{s}^{2} \mathbf{R}_{a}\right)^{-1} \mathbf{m} .
$$

Substituting (8) back into (7), we obtain that the minimal MSE is given by

$$
\mathrm{MSE}_{\mathrm{o}}=\sigma_{s}^{2}-\sigma_{s}^{4} \mathbf{m}^{H}\left(\mathbf{R}_{i+n}+\sigma_{s}^{2} \mathbf{R}_{a}\right)^{-1} \mathbf{m} .
$$

From (8), we can see that the knowledge of the signal power is required in the MMSE beamformer. However, in practice this knowledge may be difficult to obtain. To avoid this difficulty, the MLSE approach can be applied. It minimizes the LSE of the array observations and does not require any knowledge of the signal power.

Assuming that the signal waveforms are independent at different sampling times, the expectation of the LSE of a single snapshot of the array observation with respect to $\mathbf{a}$ is given by

$$
\begin{aligned}
\mathrm{LSE} & =\mathrm{E}_{\mathbf{a}}\left\{(\mathbf{y}-\mathbf{a} \hat{s})^{H} \mathbf{R}_{i+n}^{-1}(\mathbf{y}-\mathbf{a} \hat{s})\right\} \\
& =(\mathbf{y}-\mathbf{m} \hat{s})^{H} \mathbf{R}_{i+n}^{-1}(\mathbf{y}-\mathbf{m} \hat{s})+\hat{s}^{2} \operatorname{Tr}\left\{\mathbf{R}_{i+n}^{-1} \mathbf{C}\right\} .
\end{aligned}
$$

Differentiating (9) with respect to $\hat{s}$ and equating to 0 , we have that

$$
\hat{s}=\frac{1}{\mathbf{m}^{H} \mathbf{R}_{i+n}^{-1} \mathbf{m}+\operatorname{Tr}\left\{\mathbf{R}_{i+n}^{-1} \mathbf{C}\right\}} \mathbf{m}^{H} \mathbf{R}_{i+n}^{-1} \mathbf{y} .
$$

Thus, the MLSE beamformer is

$$
\mathbf{w}_{\mathrm{MLSE}}=\frac{1}{\mathbf{m}^{H} \mathbf{R}_{i+n}^{-1} \mathbf{m}+\operatorname{Tr}\left\{\mathbf{R}_{i+n}^{-1} \mathbf{C}\right\}} \mathbf{R}_{i+n}^{-1} \mathbf{m} .
$$

Substituting (10) back into (9), the minimal LSE is given by

$$
\mathrm{LSE}_{\mathrm{o}}=\mathbf{y}^{H} \mathbf{R}_{i+n}^{-1} \mathbf{y}-\frac{\left|\mathbf{y}^{H} \mathbf{R}_{i+n}^{-1} \mathbf{m}\right|^{2}}{\mathbf{m}^{H} \mathbf{R}_{i+n}^{-1} \mathbf{m}+\operatorname{Tr}\left\{\mathbf{R}_{i+n}^{-1} \mathbf{C}\right\}} .
$$

From (2), (8), and (10), it can be seen that, when the steering vector is precisely known (i.e., $\mathbf{C}=0$ ), the MSINR, MMSE, and MLSE beamformer weight vectors can be simplified to

$$
\begin{aligned}
\mathbf{w}_{\text {MSINR }} & =\tilde{\alpha} \mathbf{R}_{i+n}^{-1} \mathbf{m} \\
\mathbf{w}_{\text {MMSE }} & =\frac{\sigma_{s}^{2}}{1+\sigma_{s}^{2} \mathbf{m}^{H} \mathbf{R}_{i+n}^{-1} \mathbf{m}} \mathbf{R}_{i+n}^{-1} \mathbf{m} \\
\mathbf{w}_{\text {MLSE }} & =\frac{1}{\mathbf{m}^{H} \mathbf{R}_{i+n}^{-1} \mathbf{m}} \mathbf{R}_{i+n}^{-1} \mathbf{m}
\end{aligned}
$$


where $\tilde{\alpha}$ is an arbitrary nonzero scalar. In the case of MVDR beamformer, $\tilde{\alpha}=1 /\left(\mathbf{m}^{H} \mathbf{R}_{i+n}^{-1} \mathbf{m}\right)$. From (11)-(13) we can see that these three weight vectors differ only in their scaling factors. Therefore, when the steering vector and the signal power are exactly known, we should choose the MMSE beamformer, since it simultaneously minimizes the MSE and maximizes the SINR. When the signal power is unknown, we can use the MLSE beamformer, because it satisfies both the MLSE and MSINR criteria. Moreover, comparing (12) with (13) we have that the MLSE beamformer asymptotically minimizes the MSE in the high signal power case.

\section{PERFormance TradeOFFS}

\section{A. MMSE versus MSINR}

Comparing (8) and (2), we see that when the steering vector is random, the MMSE approach does not maximize the output SINR. That is, the MMSE and MSINR criteria can not be attained simultaneously. Since every beamformer results in a certain MSE and SINR, it is associated with a point on the MSE-SINR plane. Apparently, a beamformer providing good MSE-SINR tradeoff should yield large SINR and small MSE. However, these two objectives are contradictive to each other. Therefore, not all points on this plane are achievable.

It is of interest to develop a beamformer which provides an optimal MSE-SINR tradeoff. Such beamformer can, for example, minimize the MSE subject to some given SINR constraint. The MSE-SINR plane points associated with this beamformer will form an optimal tradeoff curve which is the frontier that divides the whole MSE-SINR plane into two parts. All points on one part of the plane are achievable, while the points on the other part can not be achieved.

Let us assume now that the signal power is known. The weight vector of a beamformer which minimizes the MSE subject to a SINR constraint can be formulated as the following optimization problem

$$
\min _{\mathbf{w}} \operatorname{MSE} \quad \text { s.t. } \frac{1}{\mathrm{SINR}} \leq \gamma
$$

where SINR and MSE are given by (1) and (7), respectively, and $\gamma$ is a given positive scalar. In order to have a feasible optimization problem, $\gamma$ should satisfy $\gamma \geq 1 /$ SINR $_{\mathrm{o}}$, where SINR $_{\mathrm{O}}$ is given by (5).

Substituting (7) and (1) into (14), we have the following optimization problem

$$
\begin{array}{ll}
\min _{\mathbf{w}} & \mathbf{w}^{H}\left(\mathbf{R}_{i+n}+\sigma_{s}^{2} \mathbf{R}_{a}\right) \mathbf{w}-2 \operatorname{Re}\left\{\sigma_{s}^{2} \mathbf{w}^{H} \mathbf{m}\right\}+\sigma_{s}^{2} \\
\text { s.t. } & \mathbf{w}^{H}\left(\mathbf{R}_{i+n}-\gamma \sigma_{s}^{2} \mathbf{R}_{a}\right) \mathbf{w} \leq 0 .
\end{array}
$$

When $\gamma=1 / \mathrm{SINR}_{\mathrm{o}}$, the problem (15) is equivalent to the design of a beamformer that minimizes the MSE under the maximal SINR constraint. The weight vector of such a beamformer can be represented as $\mathbf{w}=c \mathcal{P}\left\{\mathbf{R}_{i+n}^{-1} \mathbf{R}_{a}\right\}$, where $c$ is a nonzero scalar that should be chosen to yield the minimal
MSE. Thus, the optimal $c$ can be computed as

$$
\begin{aligned}
c_{\mathrm{opt}}= & \arg \min _{c} \mathbf{w}^{H}\left(\mathbf{R}_{i+n}+\sigma_{s}^{2} \mathbf{R}_{a}\right) \mathbf{w} \\
& -2 \operatorname{Re}\left\{\sigma_{s}^{2} \mathbf{w}^{H} \mathbf{m}\right\}+\left.\sigma_{s}^{2}\right|_{\mathbf{w}=c \mathcal{P}\left\{\mathbf{R}_{i+n}^{-1} \mathbf{R}_{a}\right\}} \\
= & \arg \min _{c} t_{1} c^{2}+t_{2} c+\sigma_{s}^{2}
\end{aligned}
$$

where

$$
\begin{aligned}
& t_{1} \triangleq \mathcal{P}\left\{\mathbf{R}_{i+n}^{-1} \mathbf{R}_{a}\right\}^{H}\left(\mathbf{R}_{i+n}+\sigma_{s}^{2} \mathbf{R}_{a}\right) \mathcal{P}\left\{\mathbf{R}_{i+n}^{-1} \mathbf{R}_{a}\right\} \\
& t_{2} \triangleq-2 \operatorname{Re}\left\{\sigma_{s}^{2} \mathcal{P}\left\{\mathbf{R}_{i+n}^{-1} \mathbf{R}_{a}\right\}^{H} \mathbf{m}\right\} .
\end{aligned}
$$

Since $t_{1}>0$, we have $c_{\mathrm{opt}}=-t_{2} / 2 t_{1}$, and

$$
\mathbf{w}=-\frac{t_{2}}{2 t_{1}} \mathcal{P}\left\{\mathbf{R}_{i+n}^{-1} \mathbf{R}_{a}\right\}
$$

When $\gamma>1 / \mathrm{SINR}_{\mathrm{o}}, \mathbf{R}_{i+n}-\gamma \sigma_{s}^{2} \mathbf{R}_{a}$ is an indefinite matrix. Therefore, in general the problem (15) is a nonconvex optimization problem. However, there always exists $\mathbf{w}$ satisfying $\mathbf{w}^{H}\left(\mathbf{R}_{i+n}-\gamma \sigma_{s}^{2} \mathbf{R}_{a}\right) \mathbf{w}<0$. Therefore, the optimization problem (15) is strictly feasible and belongs to the class of quadratic optimization problems with a quadratic constraint. It is known that these problems satisfy strong duality even if they are not convex, provided that the problem is strictly feasible [11], [12]. Hence, the solution to (15) exists if and only if there exists $\mu \geq 0$ such that [11], [12]

$$
\begin{aligned}
{\left[\mathbf{R}_{i+n}+\sigma_{s}^{2} \mathbf{R}_{a}+\mu\left(\mathbf{R}_{i+n}-\gamma \sigma_{s}^{2} \mathbf{R}_{a}\right)\right] \mathbf{w} } & =\sigma_{s}^{2} \mathbf{m} \\
\mu \mathbf{w}^{H}\left(\mathbf{R}_{i+n}-\gamma \sigma_{s}^{2} \mathbf{R}_{a}\right) \mathbf{w} & =0 \\
\mathbf{R}_{i+n}+\sigma_{s}^{2} \mathbf{R}_{a}+\mu\left(\mathbf{R}_{i+n}-\gamma \sigma_{s}^{2} \mathbf{R}_{a}\right) & \succeq 0 \\
\mathbf{w}^{H}\left(\mathbf{R}_{i+n}-\gamma \sigma_{s}^{2} \mathbf{R}_{a}\right) \mathbf{w} & \leq 0
\end{aligned}
$$

If $\gamma$ is larger than a certain threshold $\gamma_{t}$, then the inequality (19) becomes inactive, i.e., $\mathbf{w}^{H}\left(\mathbf{R}_{i+n}-\gamma \sigma_{s}^{2} \mathbf{R}_{a}\right) \mathbf{w}<0$. Then, from (17) it follows that $\mu=0$. Substituting $\mu=0$ back into (16), we obtain that such a beamformer is just the MMSE beamformer given by (8). Moreover, the threshold $\gamma_{t}$ can be obtained by substituting (8) into $\mathbf{w}^{H}\left(\mathbf{R}_{i+n}-\gamma_{t} \sigma_{s}^{2} \mathbf{R}_{a}\right) \mathbf{w}=0$, and is given by

$$
\gamma_{t}=\frac{\mathbf{m}^{H}\left(\mathbf{R}_{i+n}+\sigma_{s}^{2} \mathbf{R}_{a}\right)^{-1} \mathbf{R}_{i+n}\left(\mathbf{R}_{i+n}+\sigma_{s}^{2} \mathbf{R}_{a}\right)^{-1} \mathbf{m}}{\sigma_{s}^{2} \mathbf{m}^{H}\left(\mathbf{R}_{i+n}+\sigma_{s}^{2} \mathbf{R}_{a}\right)^{-1} \mathbf{R}_{a}\left(\mathbf{R}_{i+n}+\sigma_{s}^{2} \mathbf{R}_{a}\right)^{-1} \mathbf{m}} .
$$

When $\gamma \leq \gamma_{t}$, the beamforming vector $\mathbf{w}$ can be obtained in the following way. For the notational simplicity, let us define

$$
\mathbf{T} \triangleq \mathbf{R}_{i+n}+\sigma_{s}^{2} \mathbf{R}_{a}+\mu\left(\mathbf{R}_{i+n}-\gamma \sigma_{s}^{2} \mathbf{R}_{a}\right) .
$$

If $\mathbf{T} \succ 0$, from (16) we have that the optimal $\mathbf{w}$ is given by

$$
\mathbf{w}=\sigma_{s}^{2} \mathbf{T}^{-1} \mathbf{m} .
$$

Comparing the beamformers (8) and (21), it can be seen that they have a similar structure. In particular, the beamformer (21) has an additional term $\mu\left(\mathbf{R}_{i+n}-\gamma \sigma_{s}^{2} \mathbf{R}_{a}\right)$ with respect to (8). This term is related to the variables $\mu$ and $\gamma$, and reflects the effect of taking into account both the SINR and 
MSE criteria in our design. Moreover, when the actual steering vector is known (i.e., $\mathbf{C}=0$ ), (21) can be simplified as

$$
\mathbf{w}=\frac{\sigma_{s}^{2}}{(1+\mu)+\left(\sigma_{s}^{2}-\mu \sigma_{s}^{2} \gamma\right) \mathbf{m}^{H} \mathbf{R}_{i+n}^{-1} \mathbf{m}} \mathbf{R}_{i+n}^{-1} \mathbf{m} .
$$

It can be seen that (22) is only different from the beamformers (11)-(13) by its scaling factor.

If $\mathbf{T} \succeq 0$ (but $\mathbf{T}$ is not positive definite), then from (16) we have

$$
\mathbf{w}=\mathbf{w}_{1}+\mathbf{B w}_{2}
$$

where $\mathbf{w}_{1}=\sigma_{s}^{2} \mathbf{T}^{\dagger} \mathbf{m}, \mathbf{B}=\operatorname{null}\{\mathbf{T}\}, \mathbf{w}_{2}$ is an $(M-$ $\operatorname{rank}\{\mathbf{T}\}) \times 1$ vector, $(\cdot)^{\dagger}$ is the pseudo-inverse, and null $\{\cdot\}$ denotes the null space of a matrix. Clearly, $\mathbf{w}_{2}$ should be chosen so that (23) satisfies (17) and (19).

The remaining problem is to find the Lagrange multiplier $\mu$ in (21) and (23). It can be obtained by solving the corresponding dual optimization problem of (15). This dual problem can be written as [11]

$$
\max _{\delta, \mu} \delta \quad \text { s.t. }\left[\begin{array}{cc}
\mathbf{T} & \sigma_{s}^{2} \mathbf{m} \\
\sigma_{s}^{2} \mathbf{m}^{H} & \sigma_{s}^{2}-\delta
\end{array}\right] \succeq 0, \quad \mu \geq 0 .
$$

This is a convex semidefinite programming (SDP) problem that can be solved using modern numerical optimization software such as SeDuMi [13].

\section{B. MLSE versus MSINR}

Following the idea above, let us consider a beamformer which minimizes the LSE under the SINR constraint

$$
\min _{\mathbf{w}} \text { LSE } \quad \text { s.t. } \quad \frac{1}{\mathrm{SINR}} \leq \gamma
$$

where SINR and LSE are given by (1) and (9), respectively.

Substituting (1) and (9) into (25), we can express the latter problem as

$$
\begin{array}{ll}
\min _{\mathbf{w}} & \mathbf{w}^{H}\left(\mathbf{m}^{H} \mathbf{R}_{i+n}^{-1} \mathbf{m}+\operatorname{Tr}\left\{\mathbf{R}_{i+n}^{-1} \mathbf{C}\right\}\right) \mathbf{y} \mathbf{y}^{H} \mathbf{w} \\
& -2 \operatorname{Re}\left\{\mathbf{y}^{H} \mathbf{R}_{i+n}^{-1} \mathbf{m} \mathbf{w}^{H} \mathbf{y}\right\}+\mathbf{y}^{H} \mathbf{R}_{i+n}^{-1} \mathbf{y} \\
\text { s.t. } & \mathbf{w}^{H}\left(\mathbf{R}_{i+n}-\gamma \sigma_{s}^{2} \mathbf{R}_{a}\right) \mathbf{w} \leq 0 .
\end{array}
$$

When $\gamma>1 /$ SINR $_{\mathrm{o}}$, the problem (26) is strictly feasible and $\mathbf{w}$ is the optimal solution of (26) if and only if there exists $\mu \geq 0$ such that

$$
\begin{aligned}
{\left[a_{1} \mathbf{y} \mathbf{y}^{H}+\mu\left(\mathbf{R}_{i+n}-\gamma \sigma_{s}^{2} \mathbf{R}_{a}\right)\right] \mathbf{w} } & =a_{2} \mathbf{y} \\
\mu \mathbf{w}^{H}\left(\mathbf{R}_{i+n}-\gamma \sigma_{s}^{2} \mathbf{R}_{a}\right) \mathbf{w} & =0 \\
a_{1} \mathbf{y} \mathbf{y}^{H}+\mu\left(\mathbf{R}_{i+n}-\gamma \sigma_{s}^{2} \mathbf{R}_{a}\right) & \succeq 0 \\
\mathbf{w}^{H}\left(\mathbf{R}_{i+n}-\gamma \sigma_{s}^{2} \mathbf{R}_{a}\right) \mathbf{w} & \leq 0
\end{aligned}
$$

where

$$
\begin{aligned}
& a_{1} \triangleq \mathbf{m}^{H} \mathbf{R}_{i+n}^{-1} \mathbf{m}+\operatorname{Tr}\left\{\mathbf{R}_{i+n}^{-1} \mathbf{C}\right\} \\
& a_{2} \triangleq \mathbf{y}^{H} \mathbf{R}_{i+n}^{-1} \mathbf{m} .
\end{aligned}
$$

It can be seen from (31) that $a_{1}>0$.

Left-multiplying both sides of (27) with $\mathbf{w}^{H}$ and using (28), we have

$$
\mathbf{w}^{H} \mathbf{y}\left(a_{1} \mathbf{y}^{H} \mathbf{w}-a_{2}\right)=0 .
$$

It is obvious that $\mathbf{w}^{H} \mathbf{y} \neq 0$ as, otherwise, the beamformer output is zero. Therefore, the following equation should hold

$$
a_{1} \mathbf{y}^{H} \mathbf{w}-a_{2}=0 .
$$

Substituting (32) into (33), we obtain the following equation

$$
\mathbf{y}^{H}\left(\mathbf{w}-\frac{1}{a_{1}} \mathbf{R}_{i+n}^{-1} \mathbf{m}\right)=0 .
$$

From (34), it can be seen that the optimal w has the following form

$$
\mathbf{w}=\frac{1}{a_{1}} \mathbf{R}_{i+n}^{-1} \mathbf{m}+\mathbf{P}_{\mathbf{y}}^{\perp} \mathbf{u}
$$

where $\mathbf{u}$ is an arbitrary $M \times 1$ vector, and $\mathbf{P}_{\mathbf{y}}^{\perp}=\mathbf{I}_{M}-$ $\left(\mathbf{y}^{H} \mathbf{y}\right)^{-1} \mathbf{y} \mathbf{y}^{H}$ is the orthogonal projector onto the subspace orthogonal to that spanned by $\mathbf{y}$.

An important observation from our analysis is that, since the beamformer (35) always minimizes the LSE, we can choose $\mathbf{u}$ such that the corresponding beamformer also maximizes the output SINR. Comparing the weight vector of the MSINR beamformer of (2) with that of (35), we find that such a desired $\mathbf{u}$ exists if and only if there is a pair of $(\beta, \mathbf{u})$ such that

$$
\beta \mathcal{P}\left\{\mathbf{R}_{i+n}^{-1} \mathbf{R}_{a}\right\}=\frac{1}{a_{1}} \mathbf{R}_{i+n}^{-1} \mathbf{m}+\mathbf{P}_{\mathbf{y}}^{\perp} \mathbf{u} .
$$

Orthogonally projecting both sides of (36) onto the subspace spanned by $\mathbf{y}$ and its orthogonal subspace, respectively, we have

$$
\begin{aligned}
\frac{1}{a_{1}} \mathbf{P}_{\mathbf{y}} \mathbf{R}_{i+n}^{-1} \mathbf{m} & =\beta \mathbf{P}_{\mathbf{y}} \mathcal{P}\left\{\mathbf{R}_{i+n}^{-1} \mathbf{R}_{a}\right\} \\
\frac{1}{a_{1}} \mathbf{P}_{\mathbf{y}}^{\perp} \mathbf{R}_{i+n}^{-1} \mathbf{m}+\mathbf{P}_{\mathbf{y}}^{\perp} \mathbf{u} & =\beta \mathbf{P}_{\mathbf{y}}^{\perp} \mathcal{P}\left\{\mathbf{R}_{i+n}^{-1} \mathbf{R}_{a}\right\}
\end{aligned}
$$

where $\mathbf{P}_{\mathbf{y}}=\left(\mathbf{y}^{H} \mathbf{y}\right)^{-1} \mathbf{y} \mathbf{y}^{H}$ is the orthogonal projector onto the subspace spanned by $\mathbf{y}$. From (37), we find that $\beta$ is given by

$$
\beta=\frac{\mathbf{y}^{H} \mathbf{R}_{i+n}^{-1} \mathbf{m}}{a_{1} \mathbf{y}^{H} \mathcal{P}\left\{\mathbf{R}_{i+n}^{-1} \mathbf{R}_{a}\right\}} .
$$

Substituting (39) back into (38), we obtain

$$
\mathbf{P}_{\mathbf{y}}^{\perp} \mathbf{u}=\frac{1}{a_{1}}\left[\frac{\mathbf{y}^{H} \mathbf{R}_{i+n}^{-1} \mathbf{m}}{\mathbf{y}^{H} \mathcal{P}\left\{\mathbf{R}_{i+n}^{-1} \mathbf{R}_{a}\right\}} \mathcal{P}\left\{\mathbf{R}_{i+n}^{-1} \mathbf{R}_{a}\right\}-\mathbf{R}_{i+n}^{-1} \mathbf{m}\right]_{(40)}
$$

Finally, substituting (39) and (40) back into (35), we obtain the weight vector

$$
\mathbf{w}=\frac{\mathbf{y}^{H} \mathbf{R}_{i+n}^{-1} \mathbf{m}}{a_{1} \mathbf{y}^{H} \mathcal{P}\left\{\mathbf{R}_{i+n}^{-1} \mathbf{R}_{a}\right\}} \mathcal{P}\left\{\mathbf{R}_{i+n}^{-1} \mathbf{R}_{a}\right\} .
$$

Comparing the scalar of (39) with that of the MVDR beamformer (see (4)), we obtain that the former scalar depends on the snapshot value $\mathbf{y}$, while the latter does not. Moreover, if $\mathbf{C}=0$, then $\mathcal{P}\left\{\mathbf{R}_{i+n}^{-1} \mathbf{R}_{a}\right\}=\mathbf{R}_{i+n}^{-1} \mathbf{m}$, and in this case (41) is equivalent to the MVDR beamformer.

The beamformer (41) can be useful in the case of a random steering vector because it maximizes the output SINR and minimizes the LSE of the array observations. In fact, (41) provides a way to find the scaling coefficient of the MSINR beamformer (2) such that the LSE is also minimized. 


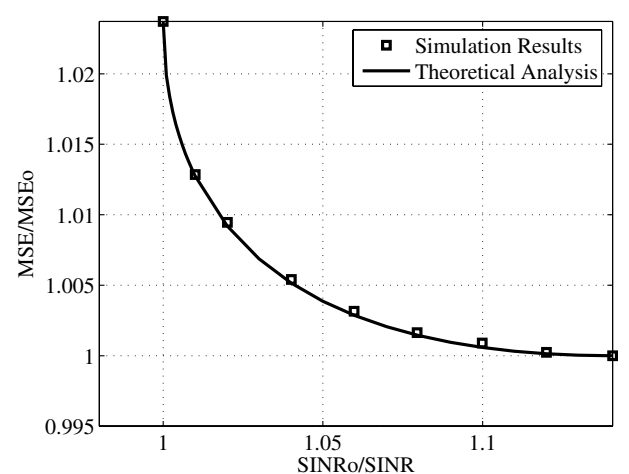

Fig. 1. MSE-SINR tradeoff with $S N R=20 \mathrm{~dB}$ and $\mathrm{INR}=0 \mathrm{~dB}$.

\section{Simulations}

Since the MLSE and MSINR criteria can be achieved simultaneously by the beamformer (35), we only need to study the tradeoffs between the MMSE and MSINR criteria. In our simulations, a uniform linear array (ULA) with $M=5$ antenna elements spaced half a wavelength apart is used. In all examples, a random SOI steering vector is assumed. Its mean vector is given by $\mathbf{m}=\left[0, e^{j \pi \sin \theta_{s}}, \cdots, e^{j(M-1) \pi \sin \theta_{s}}\right]^{T}$, where $\theta_{s}$ is the nominal direction of arrival (DOA) of the SOI that is set to be $30^{\circ}$ from the broadside of the antenna array. The interference is given by $\mathbf{i}(t)=\mathbf{a}_{i} i(t)$ where $i(t)$ is a zero mean complex Gaussian process, and $\mathbf{a}_{i}$ is the interference steering vector. We set $\mathbf{a}_{i}=\left[0, e^{j \pi \sin \theta_{i}}, \cdots, e^{j(M-1) \pi \sin \theta_{i}}\right]^{T}$ and $\theta_{i}=-30^{\circ}$ from the broadside of the antenna array.

In each example, the results are averaged over $10^{4}$ independent simulation runs. In each simulation run, the actual SOI steering vector $\mathbf{a}$ is generated by adding to $\mathbf{m}$ a complex circular Gaussian distributed random vector with zero mean the covariance matrix $\mathbf{C}=0.7 \mathbf{I}_{M}$. In each run, the MSE and the output SINR are averaged over $L=10^{4}$ data realizations and are calculated respectively as

$$
\begin{aligned}
\mathrm{MSE} & =\frac{1}{L} \sum_{t=1}^{L}\left|\mathbf{w}^{H} \mathbf{y}(t)-s(t)\right|^{2} \\
\mathrm{SINR} & =\frac{1}{L} \sum_{t=1}^{L} \frac{\left|\mathbf{w}^{H} \mathbf{a} s(t)\right|^{2}}{\left|\mathbf{w}^{H}(\mathbf{i}(t)+\mathbf{v}(t))\right|^{2}} .
\end{aligned}
$$

Throughout all our examples, we assume that the required quantities such as the interference-plus-noise covariance matrix $\mathbf{R}_{i+n}$ and the signal power $\sigma_{s}^{2}$ are exactly known. In particular, $\mathbf{R}_{i+n}$ is calculated without the presence of the signal component, and is given by $\mathbf{R}_{i+n}=\sigma_{i}^{2} \mathbf{a}_{i} \mathbf{a}_{i}^{H}+\sigma_{v}^{2} \mathbf{I}_{M}$, where $\sigma_{i}^{2}$ and $\sigma_{v}^{2}$ denote the interference and noise powers, respectively.

In the first example, we simulate a low interference-to-noise ratio (INR) scenario where the signal-to-noise ratio (SNR) is $20 \mathrm{~dB}$ and INR is $0 \mathrm{~dB}$ in a single antenna element. Fig. 1 compares the theoretical and simulated MSE-SINR tradeoff curves. The theoretical MSE and SINR are calculated using

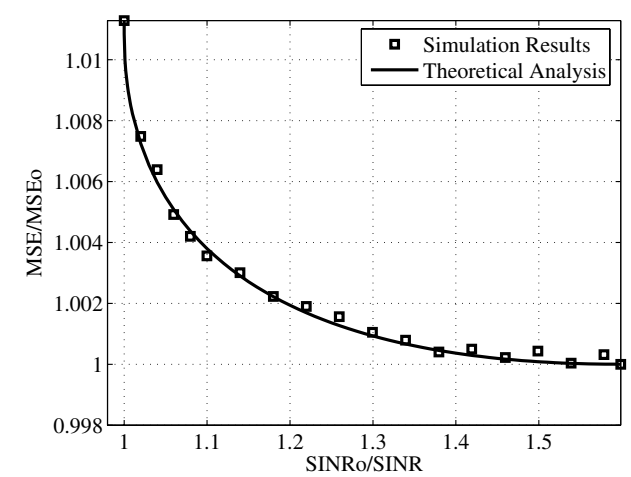

Fig. 2. MSE-SINR tradeoff with $S N R=20 \mathrm{~dB}$ and $\mathrm{INR}=15 \mathrm{~dB}$.

(7) and (1), while the simulation results are obtained using (42) and (43), respectively. The beamformer (15) has been used to obtain the simulation points.

In the second example, we consider a high INR scenario with $\mathrm{SNR}=20 \mathrm{~dB}$ and $\mathrm{INR}=15 \mathrm{~dB}$ in a single antenna element. For these parameters, Fig. 2 displays the similar curves as shown in Fig. 1. From both these figures it can be seen that our numerical and theoretical results have a very good coincidence. It is also worth noting that the shape of the tradeoff curves depends on the particular scenario parameters, such as INR.

\section{REFERENCES}

[1] H. Van Trees, Optimum Array Processing. New York, U.S.: John Wiley and Sons, 2002.

[2] Y. C. Eldar and A. Nehorai, "Mean-squared error beamforming for signal estimation: A competitive approach," in Robust Adaptive Beamforming, J. Li and P. Stoica, Eds., Wiley, 2005, pp. 259-298.

[3] H. Krim and M. Viberg, "Two decades of array signal processing research," IEEE Signal Processing Mag., vol. 13, pp. 67-94, July 1996.

[4] A. B. Gershman, "Robust adaptive beamforming," Int. J. Electron. Commun., vol. 53, pp. 305-314, Dec. 1999.

[5] O. Besson and F. Vincent, "Performance analysis of beamformers using generalized loading of the covariance matrix in the presence of random steering vector errors," IEEE Trans. Signal Processing, vol. 53, pp. 452459, Feb. 2005.

[6] O. Besson, A. A. Monakov, and C. Chalus, "Signal waveform estimation in the presence of uncertainties about the steering vector," IEEE Trans. Signal Processing, vol. 52, pp. 2432-2440, Sept. 2004.

[7] S. A. Vorobyov, Y. Rong, and A. B. Gershman, "Robust adaptive beamforming using probability-constrained optimization," Proc. IEEE Workshop on Statistical Signal Processing, Bordeaux, France, July 2005, pp. 934-939.

[8] K. L. Bell, Y. Ephraim, and H. L. V. Trees, "A Bayesian approach to robust adaptive beamforming," IEEE Trans. Signal Processing, vol. 48, pp. 386-398, Feb. 2000.

[9] S. A. Vorobyov, A. B. Gershman, and Z. Q. Luo, "Robust adaptive beamforming using worst-case performance optimization: A solution to the signal mismatch problem," IEEE Trans. Signal Processing, vol. 51, pp. 313-324, Feb. 2003.

[10] R. Lorenz and S. P. Boyd, "Robust minimum variance beamforming," IEEE Trans. Signal Processing, vol. 53, pp. 1684-1696, May 2005.

[11] S. Boyd and L. Vandenberghe, Convex Optimization. Cambridge, U.K.: Cambridge University Press, 2004.

[12] A. Beck and Y. C. Eldar, "Strong duality in noncovex quadratic optimization with two quadratic constraints," SIAM J. Optimization, submitted.

[13] J. F. Sturm, "Using SeDuMi 1.02, a MATLAB toolbox for optimization over symmetric cones," Optimization Methods and Software, vol. 11-12, pp. 625-653, Aug. 1999. 